\title{
A CASE OF SYNAESTHESIA.
}

\section{BY CHARLES S. MYERS.}

1. Introductory.

2. Colours ascribed by $A$ to tones of different pitch, sounded singly or together.

3. Conditions of A's synaesthesia.

4. Influence of timbre, loudness, and contrast of pitch upon colours.

5. A's absence of imagery.

6. Unusual features in present case.

7. 'The factor of 'sympathy.'

8. The factor of individual experience.

9. The determinants of synaesthesia generally.

1. THE subject of this note, whom for convenience we shall call $A$, is about 30 years of age. It was quite by accident that I was led to investigate his condition of synaesthesia. He happened to be one of a number of persons who were the subjects of some experiments which I was conducting upon the aesthetics of music. One part of this investigation consisted in sounding certain single tones and chords by means of tuning forks (struck with uniform loudness by an arrangement which it is unnecessary to describe here). The subject was asked what came into his mind while he was listening to individual single tones or chords; he had to describe his attitude towards it, to state if it gave him pleasure or displeasure, and, if so, to explain why.

2. The following are specimens of $A$ 's answers when single tones were sounded. The figures indicate the number of vibrations per second; the letters in brackets give the approximate musical notes.

$800\left(a^{\prime \prime} b\right)$. Gives me the idea of a bell. It is of a light-blue colour. $I$ get the idea of a fairy, then of a fairy bell. I have no visual image. It is a pleasant sound. 
$1200\left(e^{\prime \prime \prime} b\right)$. This is not so pleasant. It suggests the high note of a fiddle. It is whiter,-a silvery grey. It is too thin. I have no image of colour.

$500\left(c^{\prime \prime}\right)$. It suggests a gong. It is rosy to brown in colour. An idea of a halo comes into my mind. It is indifferent, neither pleasant nor unpleasant.

$300\left(e^{\prime} b\right)$. This recalls the chapel bell of my College. It is a deep rich brown, passing to vermilion. The sound is very pleasant, owing to its richness.

$900\left(a^{\prime \prime} \$\right)$. A blue sound,-light blue. Moderately pleasant.

$700\left(f^{\prime \prime}\right)$. It at once made me think of a pink glass finger bowl on table. I could not see the table.

(The same fork sounded again.) I call it lilac,--perhaps from association with finger bowl. It rather suggests the taste of thick soup.

Next I sounded pairs of tones simultaneously, and obtained the following answers among others:-

1000: 1200. Unpleasant. Grey-blue. No associations. Unpleasant sensations in ear.

500 : 800. Much more pleasant, a mixed colour,-blue and pinklike the Emmanuel College blazer, only the pink is deeper. The blazer suggests the river. The mixed colour is, I think, the result of the knock $^{2}$, the blue belonging rather to the knock.

400: 700. A definite mixture of two tones. I had not heard two tones in the previous sounds'. This is a combination of two colours, brown and some lighter colour, perhaps blue. It is a muddy mixture; there is confusion of the colours.

600: 1000. Distinctly blue, with an undercurrent of dull red. It is rather pleasant. I get the idea of an undercurrent.

700: 800. This is like two notes, like a light and dark blue clashing, not matching. The general effect is displeasing.

1 The forks were struck by a hammer, but later experiments showed pretty conclusively that $A$ did not attribute any colour to the knock.

$2 A$ 's failure to notice two sounds in the two previous pairs of tones is especially intereating. On another occasion I played $f^{\prime} c^{\prime \prime}$ simultaneously on the pianoforte. $A$ only observed, in addition to the lower tone, a sound of such high pitch as to be "colourless; not a musical note." On the pianoforte, on other accasions, $A$ only failed to recognize two notes in the case of the octave. 
$600: 1200$. I only hear one sound. It is a bright pink, and quite pleasant. A salmon or mullet suggests itself, probably owing to colour.

$800: 1000$. Again there is an attempt to separate a blue from grey. But I am not sure the grey is there. I think the colour is an electric blue with a sheen-like effect, which suggests the hood of a gown, the B.A. hood of Birmingham University.

600: 800. This gives a burnt-sienna effect, with an overtinge of blue. It is indifferent or unpleasant. It suggests a French artist, whose name I forget, who paints with an overtinge of blue.

800: 900. Only one sound. A good thorough mixture of colours, a steel blue colour,-as if the components were being rotated on a colour wheel.

From these answers it will be observed that a complete mixture of the colours of the individual tones only rarely occurs when they are sounded simultaneously. To test this on another occasion, I presented the "blue" tuning-fork tone 512 with the "orange" Tonmesser tone 256. But the colours would not combine; $A$ obtained both blue and orange. At the same sitting $I$ also presented to him a few single tuning-fork tones, both those I had given him singly before and those which I had already sounded simultaneously with others.

$600\left(e^{\prime \prime} b\right)$. A rich dark Prussian blue, suggesting pictures, such as Burne-Jones painted.

$500\left(c^{\prime \prime}\right)$. Soft brown. It suggests a lady's dress.

$1200\left(e^{\prime \prime \prime} b\right)$. Half-way between blue and white. It suggests a clear sky after sunset.

$500\left(c^{\prime \prime}\right)$. Brown to pink, finally shading off to blue. It appeared to change as the tone was dying away.

$300\left(e^{\prime} b\right)$. Brown with definite pinkish tinge, finally shading off to pink.

$1300\left(e^{\prime \prime \prime}\right)$. Thinnish blue.

$1200\left(e^{\prime \prime \prime} b\right)$. Fainter, thinner. Blue, shading off to grey.

$700\left(f^{\prime \prime}\right)$. This seems like a mixture of pink and blue. It reminds me again of the College blazer; only it is bluer than before.

In all these cases the colour came first, and later suggested the 
object or scene (when it occurred)1. On another occasion, I sounded three or more tones, simultaneously. The chord 300, 500,600 was described as "a medley of colours," finally distinguishable as purple. The chord 400, 600, 800 gave rise to a "brown and a colourless blue." The chord 600, 800, 1000, 1200 produced "a distinct pink and a blue, possibly two blues." The chord $300,800,1000,1200$, caused "a very definite red tinge, pink to red predominant, with other colours playing about."

Several other sittings were obtained from $A$, during which other tones and other instruments were employed.

The following table gives the series of colours obtained for the tuning-fork tones of different pitch:

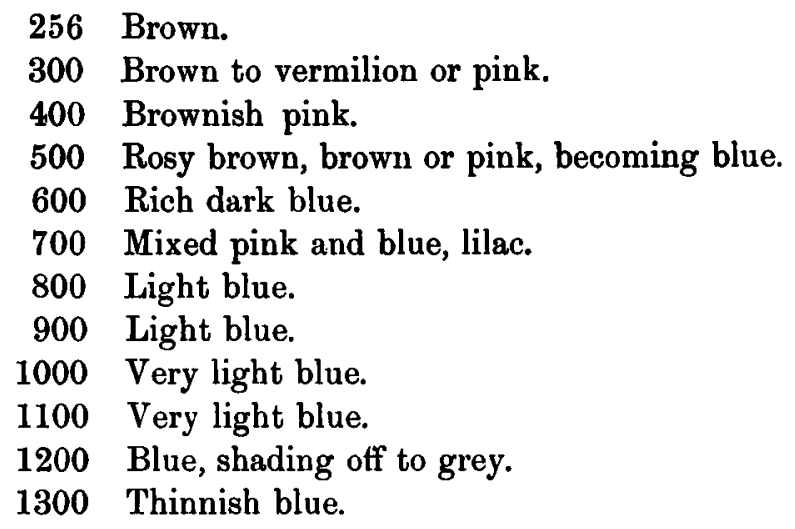

For higher tones, a Galton whistle was used. At 3000 vibrations $A$ began to note a greenish tinge in the blue. Between 4000 and 12,000 it was definitely green, but becoming more and more colourless, passing in the case of tones above 12,000 into a colourless grey.

For tones below 256, I employed an Appun's Tonmesser, obtaining from $A$ an orange or reddish orange colour for the tones in the region of 150 and 200. I also tested $A$ with the lowest tones of the pianoforte. $C_{0}$ (about 65 vibrations per second) and even $F_{0}$ were dark brown, $c^{0}$ was dark chestnut. To $C_{1} A$ found it difficult to give a colour. No sound ever appears black to him; he said, "I don't know what a black sound would be like."

We have thus obtained from $A$ a scale of colours, each of which is in some way related to a tone of a given pitch. Brown and orange

1 It is true that in some of the earliest experiments $A$ reported the colour after he had mentioned some associated object, but he is quite certain that the colour always came to him quite independently. 
colours characterise tones of a pitch below 600 vibrations per second; blue is the predominant colour of tones between 600 and 4000 vibrations, changing into green, which remains the colour of tones up to 12,000 vibrations, beyond which the tones are a colourless grey.

3. A has had this synaesthesia as long as he can remember. He does not think it has ever undergone any change. The colours are especially apt to appear when he is at a concert, but he often actively suppresses them. Words and numbers evoke no colour and no spatial schemes. Voices, on the other hand, are coloured; Madame Clara Butt's, for example, being violet, and male voices (definite instances given by $A$ ) being pink, red or brown, according to their depth and timbre. Similarly, the tones of the violoncello are brown to pink, of the bassoon brown to yellow, and of the horn brown to rose. The tones of the trombone are still redder, while the tones of the violin are pink to blue, and those of the fife are light blue verging towards green. Green, says $A$, occurs very rarely among the sounds of nature, the notes of certain birds "shading from blue through peacock colour to green."

4. But the colours which $A$ attaches to tones of different pitch are not absolute. They depend-

(a) On the timbre of the sound, as the following table shows:

Pitch
200
250
256
300
400
500
1000
1200

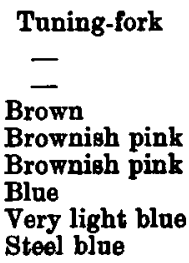

Tone variator

Dull brown

Lilac, pink

Lilac

Blue

Blue

Blue with trace of green

Peacock blue
Tonmesser

Reddish orange

Reddish pink

Light orange to pink

The richer tones take on brighter, 'higher' colours, the browns, pinks and blues of the forks becoming tinged respectively with red, blue, and green in less pure tones of the Tone variator and the Tonmesser.

(b) On the loudness of the tone. As the fork tones 'ring off,' they acquire 'higher' colours. Thus,

300 passes from brown to pink or vermilion,

$\begin{aligned} 500 " & =\left\{\begin{array}{l}\text { brown or } \\ \text { pink }\end{array} \text { to blue, }\right. \\ 1200 " & \text { blue to grey. }\end{aligned}$

This is in accordance with the well-known apparent rise of pitch in

1 Further examples are given in Table I, page 237. 
tuning-forks as their sound dies away. But $A$, it is hardly necessary to remark, was wholly unaware of such change of pitch.

(c) On the pitch of the immediately preceding tone. Thus 500, when sounded after 1200, appeared as rose-brown, and 500, when sounded after 600, appeared as soft brown. Yet 500, given on two occasions in the absence of preceding tones, was called blue, and (upon my suggesting brown) $\boldsymbol{A}$ insisted "certainly not brown or rose."

Similarly 256 , when sounded after 128 , appeared as bluish brown, but when given alone was called brown. 150, when sounded after 250, appeared as deep brown, but when given alone was called orange.

In each case, a previous lower or higher tone 'raises' or 'lowers' the colour of the following tone. This contrast effect appears to occur (but less markedly) with simultaneous as well as with successive tones.

5. It will be observed that in the introspective data given on pages 228-9 $A$ several times asserts the absence of visual imagery. On many other occasions he insisted, "I don't see the colours in my mind, I have no imagery of them." Several simple association experiments, subsequently conducted, yielded a similar result. In these experiments, such words were chosen (e.g. signal, theatre, whistle, star, band, strain, crocus, paper) as might have reasonably been expected to evoke some form of imagery. But $A$ insisted that his imagery was verbal or more often that his thoughts were entirely imageless. He had no visual, auditory or kinaesthetic imagery of the external objects thought of.

Nor were the colours of tones at all affected by the presence of actual colour before his mind. Some experiments were tried in which $A$ was instructed to think of a definite colour when the sound was produced. In other experiments he was asked to regard a sheet of given colour. But in neither case was the colour of the sound affected. As $\boldsymbol{A}$ said, "the two colours remain separate."

The degree of $A$ 's musical ability can be at once estimated by those of his introspective remarks, given on pages 229-30, which I have italicised. He states, however, that he is very fond of music, although it produces little emotional effect on him. He cannot reproduce airs he has heard, but occasionally he is worried by "tunes running in the head." $\mathrm{He}$ obtains more pleasure from pure melody than from the effects of sound colour, and finds least pleasure in the rhythmical element of music.

6. $A$ 's case is unusual in two respects. In the first place, colour synaesthesiae more usually occur in connexion with vowels, . words, persons, days of the week, months of the year, hours of the day, or languages. It is exceedingly rare to find cases where they are confined 
solely to musical sounds. Moreover, very many of those cases of synaesthesia which at first sight resembles $A$ 's, are fundamentally different. In one class of such synaesthesiae, the notes of an octave have each a different colour, and these colours are repeated in the corresponding notes of all other octaves. The colour, in fact, is attached not so much to the absolute pitch of the tone as to its name or its position in the octave. In many of these cases it appears that the synaesthesia is really derived from that of the vowel in the name of the tone. Thus in a case reported by J. Breton', we have the following correspondence between the colour of the tone and that of the vowel contained in the name of the tone:

\begin{tabular}{|c|c|c|c|}
\hline $\begin{array}{c}\text { Name of note } \\
\text { Do } \\
\text { Re' }\end{array}$ & $\begin{array}{l}\text { Colour of tone } \\
\text { Black and white } \\
\text { Brown }\end{array}$ & $\begin{array}{c}\text { Vowel sound } \\
\mathbf{0} \\
\mathbf{E}^{\prime \prime}\end{array}$ & $\begin{array}{l}\text { Colour of vowel } \\
\text { Grey-blaok } \\
\text { Colour of undyed wool (beige) }\end{array}$ \\
\hline & Fed & I & Red \\
\hline $\begin{array}{l}F_{a} \\
\text { La }\end{array}$ & Grey & $\mathbf{A}$ & Black and white \\
\hline
\end{tabular}

But even in this case there is one colour which cannot be thus explained; the note $S o l$ was red, yet we have no report that $\check{C}$ had this colour. And in another case ${ }^{2}$ though the note $a$ and the vowel $a$ and the note $e$ and the vowel $e$ were of the same colour respectively, yet other notes had colours independent of their letters and preserved their colours even when the experimenter succeeded in puzzling the subject so that he was uncertain of the name of the note. Obviously subjects of this class have, as a rule, very considerable musical ability. Their colours are usually distinctly affected by sharpening or lowering the pitch of a given note. They insist that the major chords bave a brighter colour than the minor, that the colour of a piece of music is determined by the key or by the composer ${ }^{3}$, and so on.

From this type of tone synaesthesia, $A$ 's case is clearly distinct. Nor does it coincide with another type in which association with the colour of the instrument plays a part4. There is no reason why the colour of the tones of a violin should lie between pink and blue or those of a bassoon be between brown and yellow, if the colour of the tones of different pitch had been derived from the instruments producing them.

1 Cited by Henri Laures, Les Synesthésies, Paris, 1908.

R. Lach, "Ueber einen interessaaten Spezialfall von Audition Coloree," Sammelbd. d. Internat. Musikgesell. 1902, 1v. 589.

3 Cf. Th. Flournoy, Des phenomènes de Synopsie, Paris, 1893, p. 100.

4 Ibid. p. 606. 
$A$ 's case is also unusual in the complete absence of visual imagery. The colours come to him as mere thoughts. They have no form, no position in space. He knows quite well what images are, but he gets them very rarely. This condition may be contrasted with the description given by Binet ${ }^{1}$ of the general characteristics of persons who have coloured hearing. They display, he says, a marvellous wealth of imagery in describing their mental state, they are characterised by their love for colour and for nature, by their fondness for thinking in visual imagery, by their general culture, or their literary or artistic profession. "One point," he says (p. 607), "is certain, namely that the impressions of colour suggested by definite auditory sensations are mental images." So Sokolov ${ }^{2}$ writes of these people as having a very lively imagination, deep sensibility and a visual type of memory; he states that they are incapable of working with pure abstractions, they having "always the absolutely concrete mind." Much of this is quite inapplicable to $A$ 's mental build. On the contrary, his memory is certainly not of the visual type; he is occupied constantly with abstract problems, being a very able mathematician and a person of exceptionally wide range of interests.

In many cases of synaesthesia the imagery is certainly most vivid. In one subject", for example, its vividness almost approached that of a hallucination. He localised the colour sometimes on the retina, sometimes before the lens or cornea, sometimes between the eyes! The tone gave rise to a field of colour upon which raised points, flickering, glittering and lustrous, appeared in relief. But psychology has passed beyond the stage when imagery was considered an essential element in conceptual experience. No one now questions the occurrence and the importance of imageless thought nor, among some individuals, the even complete absence of sensory imagery. We are coming to regard imagery no longer as the master, but as the servant, the bearer, of thought or meaning,--essential no doubt for mental development and persistent in concrete types of mind, but gradually becoming discarded as experience is centred more and more in the abstract.

7. I pass now to points of nore theoretical interest. For all of us, different pitches have what $I$ have elsewhere ${ }^{4}$ ventured to term different 'tone characters.' The lowest tones appear heavy, massive, thick, rounded and dull; the highest are light, fine, thin, pointed and bright;

1 "La problème de l'audition colorée," Rev. des deux mondes, 1894, cxirI. 586.

2 "L'individuation colorée," Rev. philos. 1910, LI. 41.

3 Lach, loc. cit.

4 Textbook of Experimental Psychology, 2nd edition, Cambridge, 1911, 1. 32, 33. 
and intermediate pitches have intermediate characters. The colours which $\boldsymbol{A}$ ascribes to different pitches appear to be of a similar nature. For him the deep tones are brown to pink, the highest are green to colourless, while tones of medium pitch are lilac or blue. And just as we may change at will the massiveness, the roundedness or the brightness of a pure tone by combining with it certain other pure tones (overtones) in suitable number and intensity, so $A$ 's colours may be changed by interfering with the purity of the tone stimulus. $\mathrm{He}$ ascribes lower colours to the tones of a tuning-fork than to the more metallic tones of the Tonmesser or the richer bottle tones of the Tone variator, of the same pitch. This accords with the well-known fact that a tone poor in overtones appears lower in pitch than the same tone accompanied with overtones.

Indeed $A$ may be said to some extent to hear in terms of colour. He ascribes 'higher' colours to higher sounds without being aware of an actual change in pitch (cf. page 232). He may even recognize the presence of two colours, without being aware that two tones are present (cf. page 230).

For his own part, $\boldsymbol{A}$ regards his synaesthesia as the result of some 'sympathy' existing in him between auditory and visual experiences. A similar sympathy, less developed, appears to me responsible for the tone characters we ascribe to different pitches. It is some sympathy between experiences of pitch and those of movement, touch and luminosity that makes us describe a tone as heavy, rounded or dull. The physiological and psychological bases for this sympathy are quite unknown to us; but, inasmuch as synaesthesiae seem to be commoner among children than among adults, and to arise generally (though, it appears, by no means invariably) during childhood, their origin may perhaps be ascribed to the persistence of a primitive stage in the differentiation and elaboration of sensations and in the development of their functional inter-relation.

8. That it is individual experience that determines just the colours of sounds of different timbre and pitch is indicated by the following tables', which give the colours ascribed by various subjects endowed with colour synaesthesia.

1 The letters at the head of the columns have the following references. $A$ is the case described in this paper. H is Hoffman's case described by bim in 1786, and by Goethe in his Theory of Colours in 1810. Rf is Joachim Rafi. Rs is a case related by A. de Roches. $L$ is a case of Lauret's. B and $L$ one of Bleuler and Lehmann's. $K$ is a case (Miss B.) described by W. O. Krohn from whose paper (Amer. Journ. of Psychol. 1892-3, v. 20-41) the other cases have been taken. 
TABLE I.

\begin{tabular}{|c|c|c|c|c|c|}
\hline Instrument & $\mathbf{A}$ & H & Rf & Rs & L \\
\hline Violoncello & $\begin{array}{l}\text { Brown to } \\
\text { pink }\end{array}$ & Indigo blue & - & - & $\begin{array}{l}\text { Chestnut to } \\
\text { carmine }\end{array}$ \\
\hline Horn & $\begin{array}{l}\text { Brown to } \\
\text { rose }\end{array}$ & - & Purple & - & \\
\hline Clarinet & $\begin{array}{l}\text { Yellowish } \\
\text { to bright } \\
\text { blue }\end{array}$ & Yellow & - & - & Yellow \\
\hline $\begin{array}{l}\text { Oboe } \\
\text { Violin }\end{array}$ & $\begin{array}{l}\text { Yellow } \\
\text { Pink to blue }\end{array}$ & $\begin{array}{l}\text { Rose red } \\
\text { Very bright } \\
\text { blue }\end{array}$ & Yellow & $\begin{array}{l}\text { Blue } \\
\text { Deep violet or } \\
\text { glossy black }\end{array}$ & $\begin{array}{l}\text { Chrome yellow } \\
\text { Garnet, orange, } \\
\text { yellow to white }\end{array}$ \\
\hline Flute & & Dark red & $\begin{array}{l}\text { Intense } \\
\text { azure blue }\end{array}$ & & $\begin{array}{l}\text { Yellow, blue to } \\
\text { white }\end{array}$ \\
\hline $\begin{array}{l}\text { Flageolet or } \\
\text { Fife }\end{array}$ & $\begin{array}{l}\text { Light blue } \\
\text { to green }\end{array}$ & Violet & Grey & $\begin{array}{l}\text { Deep violet or } \\
\text { glossy black }\end{array}$ & - \\
\hline Trumpet & $\begin{array}{l}\text { Scarlet to } \\
\text { bluish pink }\end{array}$ & Bright red & Scarlet & Brilliant yellow & - \\
\hline
\end{tabular}

TABLE II.

\begin{tabular}{l|c|c|c|c|c}
\hline Pitch & A & B and L & Rs & K & L \\
\cline { 2 - 5 } Low & $\begin{array}{l}\text { Brown to pink } \\
\text { Medium } \\
\text { High }\end{array}$ & $\begin{array}{l}\text { Lilac to blue } \\
\text { Green to white } \\
\text { brown } \\
\text { Yellow } \\
\text { White }\end{array}$ & $\begin{array}{l}\text { Yellow } \\
\text { Deep violet or } \\
\text { black }\end{array}$ & $\begin{array}{l}\text { Red } \\
\text { Yellow }\end{array}$ & $\begin{array}{l}\text { Wavy yellow } \\
\text { Blue to white }\end{array}$ \\
\hline
\end{tabular}

It is impossible to find more than a very broad resemblance between the colours recorded by these various observers for one and the same kind of sound. The resemblance is in the main attributable to a common experience of what we have called tone-character. The deep tones, being sombre, have dark or highly-saturated hues, the high tones, being bright, are palely coloured,-with the exception of the subject $R s$, whose colours appear to be dictated solely by the order of those in the spectrum. This brings us to another point of general resemblance, namely the progression of tones and colours pari passu according to the wave lengths of the corresponding stimuli. Colours of long wave length correspond, on the whole, to tones of long wave length; and, with few exceptions, tones of higher vibration frequency give rise to colours of higher vibration frequency.

But when we come to resemblances in detail, they are characteristically absent. To one the notes of the oboe appear rose, to anotber 
yellow, to a third blue. One subject sees a green in higher notes, another sees blue, yet another yellow. It is difficult to explain these individual variations otherwise than by accidental personal associations. As is well-known, two members of the spme family, both of whom are endowed with synaesthesia, may in some instances agree, while in others they will hotly dispute the colours of certain words or vowels. But although accidental associations may thus play a part in determining the precise colour that is to be ascribed, be it to a vowel, to a person, to the day of the week or month of the year or to a tone, the primary conditio sine quâ non of synaesthesia is that nexus, or 'sympathy' as I have called it, between auditory and visual experience.

9. From these considerations it is probable that for the full development of synaesthesia a strong tendency to a certain kind of association is requisite, - a tendency to form associations between corresponding members of two bomologous series. Clearly our own subject $A$ affords a striking example of this tendency. He associates numbers with letters. Any letter (e.g. $Y$ ) immediately tends to call up the number (25) expressing its position in the alphabet. $A$ states that he has certainly had this habit since boyhood; he believes that he developed it at about twelve years of age when, in isolation owing to an infectious illness; he amused himself by "playing about with figures." At all events he is certain that the connexion did not originate as early as that between tone and colour. If at any time now, but especially when he has nothing to think of or is fatigued, or when the letters have no meaning, he stares at a group of letters, the corresponding numbers at once come to his mind. Tendencies to associations of this type $e^{1}$, combined with the sympathy (whatever be its basis) between sound and colour, and (as a rule) with a vivid visual imagery, appear to condition the phenomena of coloured hearing.

1 Occasionally $A$ has colour experiences with tastes; these appear generally to be suggested by the colour of the tasting object. $A$ observes that while the thought of a sound may provoke its colour, the thought of a taste fails to do so. 